\title{
Hipertensión portal extrahepática: trombosis espleno-portal secundaria a déficit de proteína $\mathrm{C}$
}

\author{
P. LÓPEZ SERRANO, M. A. MARTÍN SCAPA, S. ALEMÁN VILLANUEVA, \\ M. VÁZQUEZ, L. CID GÓMEZ \\ Hospital Ramón y Cajal. Madrid
}

EXTRAHEPATIC PORTAL HYPERTENSION: PORTAL AND SPLENIC VEIN THROMBOSIS SECONDARY TO PROTEIN C DEFICIENCY

\section{RESUMEN}

La trombosis portal es la causa más común de hipertensión portal prehepática. Representa un trastorno poco frecuente, que aparece normalmente relacionado con la existencia de cirrosis, enfermedades malignas hepatobiliares o alteraciones de la coagulación, siendo estas últimas uno de los factores de riesgo más importantes.

El tratamiento anticoagulante debe emplearse en todos los casos de trombosis portal aguda y en la trombosis portal crónica cuando se identifique un factor protrombótico causal.

Presentamos el caso de un varón de 29 años, diagnosticado de hipertensión portal prehepática, secundaria a trombosis portal y esplénica, que debutó con la aparición clínica de esplenomegalia y de alteraciones de la coagulación. Tras detectar un déficit de proteína $\mathrm{C}$ se instauró tratamiento anticoagulante. Un año después el paciente no ha presentado complicaciones en relación con su patología ni con el tratamiento pautado.

PALABRAS CLAVE: Hipertensión portal. Trombosis portal. Trombosis esplénica. Déficit de proteína C.

\begin{abstract}
Portal vein thrombosis (PVT) is the most frequent cause of hypertension portal extrahepatic. It is a rare disorder an the main risk factors are cirrhosis, hepatobiliary malignancies and prothrombotic disorders, which have been identified as major risk.

Therapy with anticoagulants must to be considered in acute portal trombosis or chronic one and proven hypercoagulability.

We present the case of a twenty-nine years old patient, with extrahepatic portal hypertension secondary to portal and splenic vein thrombosis, who was diagnosed because of splenomegaly and a coagulation disorder. A protein $C$ deficiency were discovered and anticoagulation and beta-blocker therapy were iniciated. One year later the patient had not presented complications concerning to the disease or to the treatment.
\end{abstract}

KEY WORDS: Hypertension portal. Portal vein thrombosis. Splenic thrombosis. Protein C defiency.

López Serrano P, Martín Scapa MA, Alemán Villanueva S, Vázquez, M, Cid Gómez L. Hipertensión portal extrahepática: trombosis esplenoportal secundaria a déficit de proteína C. An Med Interna (Madrid) 2003; 20: 473-476.

\section{INTRODUCCIÓN}

La trombosis portal es un trastorno poco habitual, descrito por primera vez por Balfour y Stewart en el siglo XIX, siendo la causa más frecuente de hipertensión portal prehepática (1). Es normalmente diagnosticada en la infancia, existiendo una historia previa de onfalitis y/o cateterización de la vena umbilical hasta en 1/3 de los casos (1). En adultos es menos frecuente y normalmente está relacionada con la existencia de cirrosis previa, enfermedades malignas hepatobiliares o la presencia de alteraciones de la coagulación, bien intrínsecas o adquiridas $(2,3)$.

La trombosis portal se caracteriza por la ausencia habitual de síntomas al inicio del cuadro y la manifestación clínica fundamental es la hemorragia digestiva por rotura de varices esofagogástricas, siendo esta complicación la principal causa de muerte (2). El diagnostico suele realizarse tras la aparición de un sangrado por varices o durante un estudio por esplenomegalia. Actualmente la ecografía doppler permite el diagnóstico precoz, con una sensibilidad mayor al $85 \%$ de los casos, y en aquellos pacientes en los cuales la trombosis portal no sea clara, el Tac abdominal con contraste o la RM permiten el diagnóstico definitivo $(4,5)$.

Presentamos el caso de un varón de 29 años, diagnosticado de hipertensión portal prehepática secundaria a trombosis portal, que debutó con la aparición clínica de esplenomegalia y de alteraciones de la coagulación en el estudio preoperatorio para cirugía de una hernia inguinal.

Trabajo aceptado: 22 de enero de 2003

Correspondencia: P. López Serrano. C/ Inspector Juan Antonio Bueno, 2, bajo F. 28924 Alcorcón, Madrid. e-mail: dalina@ teleline.es 


\section{CASO APORTADO}

Paciente varón de 29 años de edad que es remitido a nuestro servicio desde las consultas de Cirugía General por la detección de esplenomegalia y de un trastorno de la coagulación durante el estudio preoperatorio para cirugía de una hernia inguinal. El paciente refería un ingreso 5 años atrás por dolor abdominal, junto con dudosa ictericia, detectándose durante el estudio analítico, ecográfico y endoscópico discreta elevación de transaminasas, esplenomegalia y gastritis crónica, siendo el resto de análisis normales, por lo que fue dado de alta tras la mejoría de los síntomas. El paciente posteriormente se mantuvo asintomático, refiriendo unicamente sangrado abundante tras cortes faciales, ligera astenia y sensación de distensión abdominal tras la ingesta.

En el estudio realizado en nuestro servicio destacaba en la exploración física hábito asténico y coloración pajiza de piel, sin presentar estigmas de hepatopatía crónica y en el abdomen se palpaba una esplenomegalia de 5 traveses sin hepatomegalia ni otras masas, siendo el resto de la exploración normal. En la analítica presentaba un hemograma y frotis rigurosamente normales, una bioquímica con ALT de 61 (v.n: 4-50), GGT de $71 \mathrm{mg} / \mathrm{dl}$ (v. n: 7-50), siendo el resto de parámetros de función hepática, renal y lipidograma normales, y en la hemostasia, destacaba un tiempo de protrombina del $50 \%$ junto a una cefalina de 51 segundos.

Se realizó un estudio de coagulación completo que monstraba un defecto de multiples factores de la coagulación de síntesis hepática, incluyendo factores tanto vitamina $\mathrm{K}$ dependientes como independientes (factor V, IX,XI y XII), corrigiéndose la alteración en los test de coagulación tras la administración in vitro de plasma. El tiempo de Ivy era normal. Se determinaron los niveles de las proteínas $\mathrm{C}$ y S, estando los primeros disminuidos :valores del 45 y $65 \%$ de la normalidad respectivamente (normal mayor del 60\%), los anticuerpos anticardiolipina Ig M, que estaban ligeramente aumentados $(18 \mathrm{U} / \mathrm{ml}$, rango normal hasta 15), siendo el anticuerpo antifosfolípido negativo. La cuantificación de inmunoglubulinas, el espectro electroforético y la determinación de alfa1 antitripsina, ceruloplasmina, ferritina, cobre sérico y cobre en orina de 24 horas resultaron normales, siendo el estudio inmunológico de autoanticuerpos negativo.

Se realizaron serologías a virus de la hepatitis B, hepatitis C, hepatitis A, citomegalovirus, virus de Ebstein-Barr, Yersinia y Leishmania, siendo todos negativos.

En el estudio por imagen, la radiografía de torax estaba dentro de la normalidad y en la ecografía doppler abdominal se apreciaba un hígado discretamente homogéneo, sin LOES, una esplenomegalia gigante, que desplazaba el riñón izquierdo y mínima ascitis periesplénica. La vena porta no se visualizaba, ni siquiera con la utilización de potenciadores de señal, y existía una imagen de hepatización a nivel hiliar compatible con trombosis portal. La vena esplénica tampoco era visualizada, siendo compatible con trombosis esplénica concomitante, y la vena renal izquerda presentaba un calibre muy aumentado, concordante con la existencia de un shunt esplenorrenal que no pudo evidenciarse de forma clara. El sistema biliar y las venas suprahepáticas eran normales. Estos hallazgos se corroboraron en un TAC abdominal helicoidal.

Ante la existencia de datos compatibles con hipertensión portal de causa prehepática y también de posible enfermedad intrínseca hepática se decidió la realización de una laparoscopia diagnóstica con biopsia hepática. En ella el hígado presentaba una superficie lisa, de borde fino y dibujo lobulillar conservado. En la biopsia se identificó un tejido hepático con ectasias sinusoidales reactivas, sin fibrosis y con arquitectura conservada.

Se realizó una endoscopia oral donde se detectaron varices de calibre II-III en esófago distal y gastropatía congestiva incipiente, sin varices gástricas.

El paciente fue diagnosticado según los resultados de las pruebas complementarias mencionadas de hipertensión portal de causa prehepática debido a la trombosis del eje espleno-portal, probablemente secundaria al estado procoagulante por déficit de proteína $\mathrm{C}$.
Se recomendó profilaxis primaria de hemorragia por varices con nadolol, a dosis ajustadas según frecuencia cardiaca y anticoagulación oral con dicumarínicos $\left(\right.$ Sintron $\left.^{\circledR}\right)$, manteniendo INR entre cifras de 2 y 3 .

El paciente actualmente sigue revisiones en nuestras consultas, manteniéndose estable y no habiendo presentado complicaciones derivadas de su hipertensión portal o del tratamiento pautado.

\section{DISCUSIÓN}

La obstrucción de la vena porta puede surgir secundaria a uno de los siguientes mecanismos: trombosis, invasión tumoral o compresión extrínseca por una neoplasia adyacente (2).

Clínicamente, la trombosis portal (TP) representa la forma pura de obstrucción de la vena porta. La etiología es diversa y no en todos los casos se llega a identificar la causa. Los mecanismos implicados con más frecuencia son la existencia de factores trombofílicos, que se detectan hasta en el 60-75\% de los casos, y de factores locales, que aparecen en el $40 \%$ de todas las TP $(3,4,6)$.

Dentro de las alteraciones de la coagulación, existen numerosos trastornos protrombóticos que aumentan el riesgo de sufrir una TP (Tabla I) $(2-4,7,8)$. Algunos son infrecuentes, con una prevalencia inferior a 4 por $1000 \mathrm{y}$ asociadas a alto riesgo de trombosis (mayor del 10\%), como son el déficit de proteina $\mathrm{C}$, el déficit de proteína $\mathrm{S}$ y el déficit de antitrombina y otras son más comunes, con una frecuencia alrededor del $2 \%$ de la población, y asociadas a un riesgo de trombosis menor (entre el 2 y el 8\%), como son la mutación del factor $\mathrm{V}$ de Leiden y la mutación del factor II G20210.

Otras patologías adquiridas también pueden dar lugar a un estado protrombótico, algunas menos comunes, como los síndromes mieloproliferativos primarios, el síndrome antifosfolípido o la hemoglobinuria paroxística nocturna, y otras más

\section{TABLA I}

TRASTO RNOS PRO TRO M BÓ TICOS HEREDITARIOS Y ADQ UIRIDOS RELACIO NADO S CO N LA TRO M BO SIS DE LA VENA PORTA

Trastornos protrombóticos hereditarios:

Poco frecuentes (asociados a alto riesgo de trombosis)

Déficit de antitrombina

Déficit de proteína $C$

Déficit de proteína $\mathrm{S}$

Trastornos más comunes

M utación del factor $\mathrm{V}$ de Leiden

M utación del factor II G20210

Trastornos adquiridos:

Poco frecuentes (asociados a alto riesgo de trombosis): Síndromes mieloproliferativos primarios

Síndrome antifosfolípido

Hemoglobinuria paroxística nocturana

O tras condiciones más comunes:

Anticonceptivos orales

Embarazo y postparto

Estados inflamatorios o tumorales (pancreatitis, enfermedad inflamatoria intestinal, enfermedad de Behçet's, esclerodermia, lupus eritematoso sistémico)

Hiperhomocistinemia 
frecuentes, como el uso de anticonceptivos orales, la hiperhomocisteinemia, el embarazo y el puerperio.

Con respecto a los factores locales $(2,4)$ (Tabla II), las enfermedades hepatobiliares pueden dar lugar a una disminución del flujo local en la vena porta y por ello favorecer la trombosis. Las infecciones abdominales pueden también producir una trombosis portal, y el riesgo es especialmente alto si existe sepsis, deshidratación o coagulación intravascular diseminada. Los traumatismos o los procedimientos quirúrgicos abdominales también se han puesto en relación con la TP, así como la quimioembolización de tumores hepáticos, el transplante hepático o la esclerosis de varices esofágicas. Por lo tanto, en todo paciente con TP se deberá descartar la existencia de un factor protrombótico causal, incluso cuando un factor local sea identificado, siendo también cierta la aseveración contraria $(2-4,9-11)$.

La historia natural de la trombosis portal es poco conociTABLA ॥

FACTO RES LO CALES RELACIO NADOS CO N LA TRO M BO SIS DE LA VENA PORTA

\begin{tabular}{l}
\hline Factores locales inflamatorios \\
Apendicitis \\
Diverticulitis \\
Pancreatitis \\
Colecistitis \\
Abceso hepático \\
Sepsis \\
O nfalitis \\
Factores mecánicos o intervenciones abdominales \\
Traumatismo abdominal \\
Cirugía hepatobiliar \\
Esplenectomía \\
TIPS \\
Escleroterapia endoscópica de varices \\
Q uimioembolización hepática \\
Trasplante hepático \\
Cirrosis \\
Cáncer hepatobiliar \\
Fibrosis periportal \\
Hiperplasia nodular regenerativa \\
Fibrosis septal \\
Disminución del flujo portal \\
Cáncer de órganos abdominales
\end{tabular}

da. El curso clínico suele estar en relación principalmente con la enfermedad de base que produjo la trombosis y/o con las manifestaciones de la hipertensión portal. En una revisión reciente de Janssen et al (12) la mortalidad al año fue del $30 \%$ y a los 5 años del 37\%, sin embargo la cuasa de la muerte estaba relacionada con la TP en sólo la mitad de los casos. Las variables que están más asociadas a una reducción de la supervivencia son la mayor edad, la existencia de patología maligna, la cirrosis, la presencia de varices esofágicas, la hemorragia por hipertensión portal, la presencia de ascitis y la trombosis mesentérica asociada. Separando aquellos pacientes con TP no maligna, la edad, la cirrosis y la trombosis mesentérica son los factores independientemente asociados con un peor pronóstico.

Con respecto a la morbilidad de estos pacientes la compli- cación más frecuente es el sangrado por varices esofago-gástricas, con una incidencia del $17 \%$ por paciente y año. El tamaño de las varices esofágicas es el principal factor predictor de sangrado (2), sin embargo, en comparación con la hemorragia digestiva secundaria a cirrosis hepática, los pacientes con TP tienen una marcada mayor supervivencia, que parece explicada por la normalidad en la función hepática.

El diagnóstico de la trombosis portal es realizado habitualmente mediante una ecografía Doppler, que tiene una sensibilidad en trombosis moderada-severa de hasta el $100 \%$, con un $13 \%$ de falsos positivos. En los casos dudosos la realización de un TAC abdominal helicoidal o una resonancia magnética nos facilitará el diagnóstico (4). El siguiente paso tras identificar la trombosis será determinar la antigüedad del trombo, siendo la tomografía computerizada la técnica más útil para ello. Son indicativos de trombosis reciente la existencia de un trombo visible dentro de la luz portal, la ausencia de circulación colateral o la presencia de contenido intraluminal hiperintenso previo a la inyección de contraste. La presencia de cavernomatosis traduce una trombosis antigua $(4,5)$.

En el caso de que nos encontremos ante una trombosis portal aguda es posible conseguir una repermeabilización completa o extensa con tratamiento anticoagulante, bien con heparina sódica o con heparinas de bajo peso molecular. Se aconseja el inicio con heparina sódica y el posterior paso a dicumarínicos durante 6 meses $(4,7,13,14)$, manteniéndose después de este tiempo si un componente protrombótico ha sido identificado.

Cuando la trombosis portal es antigua, el tratamiento dependerá de la existencia de síntomas asociados a la hipertensión portal y de los factores predisponentes encontrados. En aquellos pacientes en los que exista riesgo de sangrado secundario a varices esofágicas, como era el caso de nuestro paciente, deberemos añadir tratamiento médico con fármacos beta-bloqueantes (propanolol o nadolol). La experiencia recogida de diferentes autores $(2-4,7)$ señala que ésta debe ser la primera línea de tratamiento, representando la terapia endoscópica la segunda opción, que permite la prevención de la recurrencia del sangrado digestivo $(2-4,7,15)$. Es relevante destacar cómo el descenso del flujo esplácnico durante el sangrado digestivo por hipertensión portal, junto con la vasoconstricción de dicho territorio por las drogas vasoactivas, podría teóricamente empeorar la situación y favorecer la extensión y/o la recurrencia de la trombosis, con el consiguiente riesgo (2); por ello, se considera de elección el tratamiento endoscópico frente al vasoactivo durante la hemorragia digestiva.

Existen evidencias sobre una interesante relación riesgo/beneficio de la anticoagulación en pacientes con trombosis portales antiguas. Janssen apunta sobre la terapia anticoagulante que ésta no incrementa ni el riesgo ni la severidad del sangrado, y si previene nuevos episodios de trombosis (15). Debemos plantearnos la terapia anticoagulante sin demora en aquellos pacientes con trombofilia filiada y con bajo riesgo de sangrado, es decir, menos de 50 años, varices esofágicas ausentes o de pequeño tamaño y ausencia de lesiones potencialmente sangrantes a otros niveles $(2,12)$. En el caso presentado se identificó un déficit de proteínas $\mathrm{C}$, por lo que se indicó el tratamiento anticoagulante, sin que hayan existido complicaciones derivadas de éste ni datos de nuevas trombosis desde entonces. Es relevante destacar el hecho de que pue- 
dan existir alteraciones de la hemostasia en los pacientes con hipertensión portal extrahepática en ausencia de lesión hepática, como ocurría en nuestro caso. La más importante es un bajo grado de coagulación vascular diseminada, que parece secundaria a la presencia de shunts portosistémicos (2).

Con respecto al lugar de la cirugía y el tipo de tratamiento quirúgico, éstos continúan actualmente a debate $(2,4,7)$. El shunt quirúrgico, que descomprimiría el sistema venoso portal de manera efectiva, podría aparecer como una opción atractiva, ya que permitiría también un uso más general de la anticoagulación $(16,17)$. Sin embargo, varios factores interfieren en el éxito de ésta opción, como son el alto riesgo de trombosis del shunt y la alta frecuencia de trombosis de otras venas, que dificulta la cirugía. Por todo ello la alternativa quirúrgica debe reservarse para aquellos casos en los que el tratamiento endoscopico falle o exista sangrado por varices no accesibles a dicha terapia $(16,17)$. Será de elección la realización de un shunt esplenorrenal distal.

El shunt transyugular intrahepático (TIPS) supone otra posibilidad terapéutica que nos permitiría eliminar el proble- ma de hipertensión portal (18), sin embargo, la experiencia es escasa y el riesgo de trombosis es incluso mayor que con la opción quirúrgica, por lo que no existe bibliografía suficiente para su recomendación.

El transplante hepático se considera el tratamiento de elección en aquellos pacientes en los que existan complicaciones secundarias a la hipertensión portal e insuficiencia hepática; en aquellos con función hepática normal representa una alternativa a las técnicas de derivación. Es de elección también en aquellos pacientes en los que esta última opción haya fallado (18).

Por lo tanto, ante un paciente con trombosis portal, si ésta es reciente, deberemos instaurar tratamiento anticoagulante. Cuando la trombosis es antigua es prioritario investigar factores protrombóticos susceptibles de correción mediante terapia anticoagulante y pautar tratamiento preventivo de sangrado por varices si éstas existieran en la endoscopia oral. El tratamiento quirúrgico no está indicado de manera preventiva y sólo cuando las complicaciones no puedan ser resueltas mediante otras alternativas no invasivas.

\section{Bibliografía}

1. Yamada RM, Antunes M, Cardoso SR, Servidoni M de F, Hessel G. Portal vein thrombosis in children: clinical and laboratory study of 26 cases. Arq Gastroenterol 1999; 36: 49-53.

2. Valla DC, Condat B. Portal vein thrombosis in adults: pathophysiology, pathogenesis and management. J Hepatol 2000; 32: 865-871.

3. P Condat B, Pessione F, Hillaire S, Denninger MH, Guillin MC, Poliquin M, Hadengue A, Erlinger S, Valla D. Current outcome of portal vein thrombosis in adults: risk and benefit of anticoagulant therapy. Gastroenterology 2001; 120: 490-497.

4. Janssen HL. Changing perspectives in portal vein thrombosis. Scand J Gastroenterol Suppl 2000; 32: 69-73.

5. Mehmet Ali Yerdel, Bridget Gunson, Darius Mirza, Kaan Karayalçin, Simon Olliff, John Buckels, David Mayer, Paul McMaster, and Jacques Pirenne. Portal vein thrombosis in adults undergoing liver transplantation. Transplantation 200; 69: 1873-1881.

6. Rosendaal FR. Venous thrombosis: a multicausal disease. Lancet 1999; 353: 1167-1173.

7. Condat B, Valla D. Management of portal vein thrombosis. Gastroenterol Clin Biol 1999; 23: 1210-1214.

8. Robson SC, Kahn D, Kruskal J, Bird AR, Kirsch RE. Disordered hemostasis in extrahepatic portal hypertension. Hepatology 1993; 18: 853-857.

9. Denninger MH, Chait Y, Casadevall N, Hillaire S, Guillin MC, Bezeaud A, Erlinger S, Briere J, Valla D. Cause of portal or hepatic venous thrombosis in adults: the role of multiple concurrent factors. Hepatology 2000; 31: 587-591.

10. Valla DC. Portal vein thrombosis and prothrombotic disorders. J Gastroenterol Hepatol 1999; 14: 1051-2052.

11. Valla D. Venous thrombosis in gastroenterology. J Mal Vasc 1991; 16: 243-248.
12. Janssen HL, Meinardi JR, Vleggaar FP, van Uum SH, Haagsma EB, van Der Meer FJ, van Hattum J, Chamuleau RA, Adang RP, Vandenbroucke JP, van Hoek B, Rosendaal FR. Factor V Leiden mutation, prothrombin gene mutation, and deficiencies in coagulation inhibitors associated with Budd-Chiari syndrome and portal vein thrombosis: results of a case-control study. Blood 2000; 96: 2364 2368 .

13. Bertrand Condat, Fabienne Pessione, Marie Helene Denninger, Sophie Hillaire, Dominique Valla. Recent Portal or Mesenteric Venous Thrombosis: Increased Recognition and Frequent Recanalization on Anticoagulant Therapy. Hepatology 2000: 32; 466-470.

14. Schafer C, Zundler J, Bode JC. Thrombolytic therapy in patients with portal vein thrombosis: case report and review of the literature. Eur J Gastroenterol Hepatol 2000; 12: 1141-1145.

15. Shun A, Delaney DP, Martin HC, Henry GM, Stephen M. Portosystemic shunting for paediatric portal hypertension. J Pediatr Surg 1997; 32: 489-493.

16. López Saiz A, Vila Carbo JJ, Fernández MS, Muro D, Ibánez V, Gutiérrez C, Roca A, Garcia-Sala C.Our experience in portal cavernomatosis in childhood: cross-sectional study and analysis of results. Cir Pediatr 1997; 10: 90-92.

17. Kato T, Romero R, Koutouby R, Mittal NK, Thompson JF, Schleien CL, Tzakis AG. Portosystemic shunting in children during the era of endoscopic therapy: improved postoperative growth parameters. J Pediatr Gastroenterol Nutr 2000: 30: 419-425.

18. Ganger DR, Klapman JB, McDonald V, Matalon TA, Kaur S, Rosenblate H, Kane R, Saker M, Jensen DM. Transjugular intrahepatic portosystemic shunt (TIPS) for Budd-Chiari syndrome or portal vein thrombosis: review of indications and problems. Am J Gastroenterol 1999; 94: 603-608. 